\title{
Scanning Tunneling Spectroscopy of Molecules on Insulating Films
}

\author{
Jascha Repp ${ }^{\star a}$ and Gerhard Meyer ${ }^{\mathrm{b}}$
}

\begin{abstract}
Ultrathin insulating films on metal substrates are unique systems for using a scanning tunneling microscope (STM) to study the electron transport properties in the weak-coupling limit. The electronic decoupling provided by the films allows the direct imaging of the unperturbed molecular orbitals, as will be demonstrated in the case of individual pentacene molecules. The coupling between electronic states localized on the adsorbate and optical phonons in a polar insulator has two important implications: Peaks in conductance spectra resulting from resonant tunneling into electronic states of the molecules are significantly broadened by the presence of the insulator. Second, the ionic relaxations in a polar insulator may lead to an interesting charge bistability in atoms and molecules. STM-based molecular manipulation has been used to form a metallo-organic complex as well as to switch the position of the two hydrogen atoms in the inner cavity of single free-base naphthalocyanine molecules.
\end{abstract}

Keywords: Insulating film $\cdot$ Molecular electronics $\cdot$ Scanning tunneling microscopy $\cdot$ Single molecule

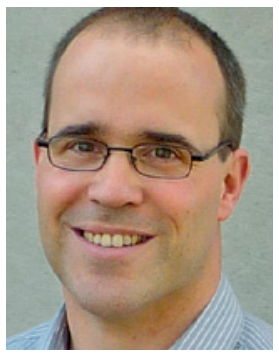

Jascha Repp worked during his $\mathrm{PhD}$ studies in the group of Prof. Dr. Karl-Heinz Rieder at the Free University in Berlin in the field of lowtemperature scanning tunnelling microscopy. In 2002 he received his $\mathrm{PhD}$ and joined the group of Dr. Gerhard Meyer at the IBM Zurich Research Laboratories, where he worked for a PostDoc for five years. Since 2007 he holds a Lichtenberg Professorship at the University of Regensburg.

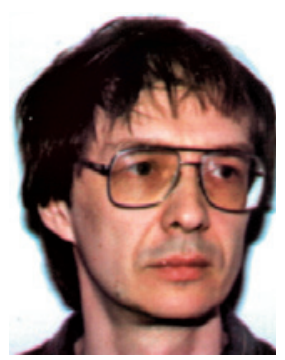

Gerhard Meyer received his $\mathrm{PhD}$ at the University Hannover, working on high-resolution lowenergy electron diffraction. Then he became a PostDoc at the IBM Research Center in Yorktown Heights, where he had the opportunity to work on scanning probe microscopy. Af-

\footnotetext{
${ }^{\star}$ Correspondence: Prof. Dr. J. Repp ${ }^{a}$

Tel.: +49 941 943-4201

Fax: +49 941 943-2754

E-mail: jascha.repp@physik.uni-regensburg.de

alnstitute of Experimental and Applied Physics,

University of Regensburg

93040 Regensburg, Germany

bIBM Research - Zurich

$\mathrm{CH}-8803$ Rüschlikon
}

ter three years he joined the group of Prof. Dr. Karl-Heinz Rieder at the Free University of Berlin, where he worked for eight years as a scientific assistent. In 2000 he accepted an offer for a research position at the Paul-Drude-Institute in Berlin. Finally in 2002 he became research staff member at the IBM Zurich Research Laboratories, where he has been working since.

\section{Introduction}

During the last decade scanning tunneling microscopy (STM) and spectroscopy (STS) has become more and more important for the field of molecular electronics, in which charge transport through single-molecule and atomic-scale structures is studied. The main drawback of STM and STS as compared to other techniques in the field is certainly the lack of an additional gate electrode that allows one to shift the molecular states with respect to the chemical potentials of the two contacts. On the other hand, the most important strength of the techniques lies in the ability to analyze the structural environment of the molecule under consideration on the atomic length-scale and to subsequently probe the electronic properties of this individual, well-characterized structure. In the past it has become very clear that the charge transport on the atomic/molecular scale is determined by the entire junction structure including the connecting electrodes, and that small changes in the atomistic arrangement of the junction may have a large influence on its characteristics. This highlights the tremendous possibilities that lie in the study of charge-transport phenomena in atomic-scale structures by means of STM and STS and explains their growing relevance in the field.

In electron transport through individual molecules three energy scales are most relevant, namely the energy separation between the molecular levels, the Coulomb charging energy $U$ and the coupling strength $\Gamma$ between the molecule and the leads. The relative ratio of these energies determines the different regimes of transport. The levels spacing and Coulomb energy are primarily determined by the molecule itself and can only be varied over roughly one order of magnitude. The coupling strength to the leads on the other hand can vary over several orders of magnitude depending on the experimental setup and therefore usually determines the transport regime.

STM and STS have recently contributed to molecular electronics both in the weak and in the strong coupling limit. As STM relies on a finite conduction of the sample, a metallic or semiconducting substrate is the natural choice. A molecule lying on such a single crystal surface with well-defined orientation can then be contacted with the STM tip at a known lateral position of the molecule. ${ }^{[1-3]}$ In these experiments one typically has access to the strong or intermediate coupling regime.

Here, we focus on the weak coupling regime. Between tip and molecule the weak coupling regime is simply achieved by the vacuum gap. To reduce the coupling between molecule and substrate however, an ultrathin insulating layer is introduced that decouples the molecule electronically from the substrate. Ultrathin refers to a thickness of only few atomic layers. For 
such a thin insulating film on a metallic substrate, electrons can still tunnel through the films, facilitating imaging by means of STM at a low tunneling current. ${ }^{[4-8]}$ On the other hand, a double layer of a wide band gap insulator is sufficient to significantly decouple an adsorbate from the metallic substrate.

This electronic decoupling in STM studies on adsorbates has opened up a new fascinating field in atomic-scale science. Examples of new physical insights and phenomena discovered in such studies are the charge control of adatoms, ${ }^{[9]}$ single-atom spin-flip spectroscopy, ${ }^{[10]}$ and vibronic spectroscopy and bipolarity in single-molecule transport.[11,12] The insulating properties of the films also enable the direct imaging of the apparently unperturbed molecular orbitals by means of STM. ${ }^{[13]}$ In this article we will review our recent STM studies on insulating films in the context of molecular electronics.

\section{Experimental Setup and Substrate Surfaces}

The experiments discussed here were carried out in ultra-high vacuum with a low-temperature STM operated at temperatures of $T=5-13 \mathrm{~K}$. As substrates we used flat as well as intrinsically stepped copper single-crystal samples. These single-crystal samples were cleaned by several sputtering and annealing cycles. Bias voltages refer to the sample voltage with respect to the tip. As STM tips we used electrochemically etched tungsten wire. For differential conductance $(\mathrm{d} I / \mathrm{d} V)$ spectroscopy, the bias voltage was modulated and the $\mathrm{d} I / \mathrm{d} V$ signal was detected by using the lock-in technique. Individual adsorbates were adsorbed at a sample temperature of 5 to $10 \mathrm{~K}$ with the sample located in the STM.

An essential prerequisite for STM studies of adsorbates on insulating films is the ability to grow stable and atomically thin insulating films on metal surfaces with a well-characterized and ordered geometric structure. For such studies a range of insulating materials has been deployed. ${ }^{[11,13-19]}$ The choice of an appropriate substrate has several other aspects: For the detection of spin transitions in such a setup, as first demonstrated by Heinrich et al.,[10] one apparently needs an intermediate coupling regime. ${ }^{[20]}$ For this purpose monolayer islands of nitrides or oxides are desirable. For our studies we were most interested in structurally very simple model systems as insulators and therefore chose alkali halide and xenon layers. As will be discussed in this article, it turned out that the mere presence of the insulator leads to a phonon-broadening of the spectroscopy data in STS. Therefore the insulator material is decisive for the energy resolution of the data. By varying the metallic substrate underneath the insulating layer one may change the work function of the system. This has significant implications as it changes the chemical potential of one of the electrodes relative to the molecular levels. As in STS usually no gate is available, this is an important alternate experimental parameter and can even be used to charge a molecule.

In addition to these considerations, the metal substrate can also be used to determine the charge state of the molecule or - more generally - the adsorbate, ${ }^{[21]}$ as follows. The well-known $\mathrm{Cu}(111)$ surface-state band survives $\mathrm{NaCl}$ or xenon adsorption and forms an interface state band that is confined to the insulator/metal interface. ${ }^{[21-23]}$ The interface state manifests itself in STM images as the typical standing-wave patterns ${ }^{[24,25]}$ arising from the scattering of the electrons. To infer the charge state from observations related to the interface state, it is important to note that the interface-state probability distribution falls off exponentially within the insulating adlayer, such that at the insulator-to-vacuum interface it is negligibly small. Consequently, the short-range potential of a neutral adsorbate should be a weak scatterer of the interfacestate electrons. A charged adsorbate, however, results in a long-range electrostatic potential that should strongly scatter the interface-state electrons below. A positively charged adsorbate, in addition, will create a local attractive potential for the interface-state electrons underneath. In general, whenever a two-dimensional, freeelectron-like interface band encounters an attractive potential, it will form a bound state, an interface-state localization. ${ }^{[21,26,27]}$ Thus, the charge state of any defect or adsorbate can unambiguously be determined to be neutral, negative, or positive, depending on the interaction with the interface state.

\section{Charge Bistability of Adatoms and Molecules}

The presence of the insulating film in the experimental setup allows for a charge bistability of adsorbates as will be discussed in the following. Whereas this was first observed for adatoms, namely individual gold atoms, the same mechanism may also lead to charge bistability in molecules. ${ }^{[28]}$ Charge bistability in molecular electronics may be interesting for memory devices. As charging a molecule affects all the states, the transport characteristics may be completely changed by changing the charge state. In addition, this allows more complex functionality to be implemented based on such a charge state switch. As the first observation of the charge state con- trol[9] was with gold adatoms we briefly describe this observation first, before discussing the implications for molecular electronics.

The charge-state control of individual gold adatoms on $\mathrm{NaCl} / \mathrm{Cu}(111)$ was done by positioning the STM tip above an $\mathrm{Au}$ adatom and applying a voltage pulse. This way the adatom can be reversibly switched between the neutral and negative charge state. Most importantly, both states are stable; that is, the additional electron will remain on the adsorbate until it is removed by a voltage pulse of reversed sign. The different charge states were identified from several observations. Experimentally, we exploited the interface-state based technique as described at the end of the previous section to determine the charge states of both species. Second, a comparison of the STM images to detailed density-functional-theory-based image simulations corroborated this analysis. Third, in a later publication, we inferred the different charge states from scanning force spectroscopy measurements. ${ }^{[29]}$ But what is the mechanism behind this charge bistability? The lateral position of the adatom did not change upon charging. A simple electron transfer without any lasting changes of the ion core positions would not be stable, because an electron residing in an excited state of the Au adatom would rapidly tunnel into the metal. The two states must therefore be associated with two different geometric configurations of the adatom and the $\mathrm{NaCl}$ film.

Density-functional theory simulations ${ }^{[9]}$ provided deeper insight into the underlying principle of the experimentally observed charge bistability: Whereas the gold atom in its neutral state leaves the ionic positions within the $\mathrm{NaCl}$ film relatively unperturbed, the negatively charged gold adatom induces strong ionic relaxations within the $\mathrm{NaCl}$ film. This relaxation pattern creates an attractive potential for the additional charge on the Au adatom, which is further stabilized by the screening charge in the metal substrate and by the electronic polarization of the ionic layer. This shifts a previously unoccupied state such that it is below the Fermi level and becomes occupied.

With that the important ingredients for such a charge bistability are identified: One needs an atomic/molecular state that is relatively close to the Fermi level. Second, the ionic relaxations resulting from a charging need to be so strong that the energetic shift associated with these relaxations is large enough to shift the corresponding state across the Fermi level. For the latter it is important to note that this energetic shift is usually larger the more the charge is spatially localized. This renders the occurrence of a charge bistability in an atom much more 


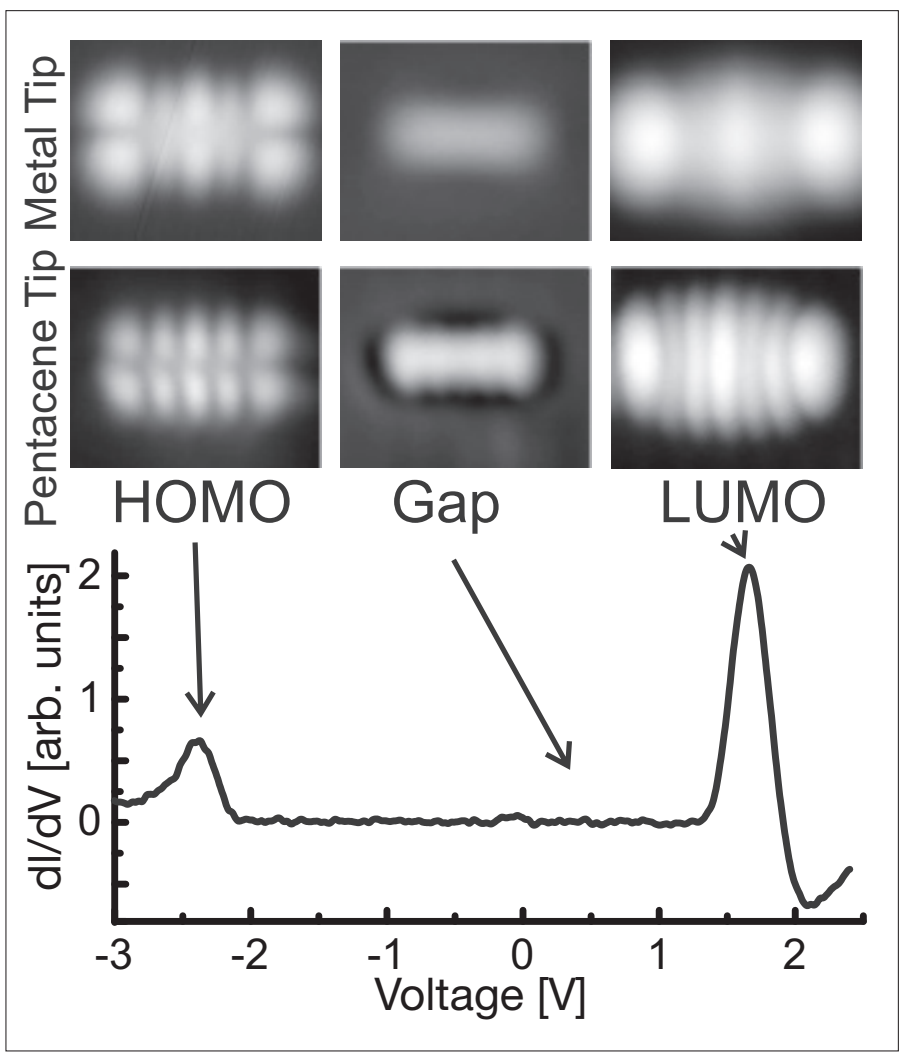

Fig. 1. Bias-dependent STM images acquired with a metal (upper row) and a pentacene-terminated tip (lower row) in relation to the peaks and gap region seen in $\mathrm{d} / / \mathrm{d} V$ spectroscopy at the center of a pentacene molecule on $\mathrm{NaCl}$. The $\mathrm{d} / / \mathrm{d} V$ signal exhibits two distinct peaks that can be attributed to the negative and the positive ion-resonance states, and a broad gap of low conductance between the peaks. Note that the STM images shown are not $\mathrm{d} / \mathrm{d} V \mathrm{maps}$, but conventional constant-current images. Adapted from ref. [13] (from left to right, upper to lower: 2300 mV, 15 pA; 429 mV, 28 pA; 1700 mV, 15 pA; 2523 mV, 22 pA; 500 mV, $22 \mathrm{pA}$; and $1793 \mathrm{mV}, 9.6 \mathrm{pA}$ )

likely than in the case of molecules. Howev$\mathrm{er}$, in molecules intramolecular relaxations may also contribute significantly to charge stabilization. In addition, the relaxation energy naturally depends on the properties of the insulator that is used. The other ingredient, namely having an electronic state close to the Fermi level, is controlled by the electron affinity (ionization potential) of the adsorbate but also by the work function of the substrate system. This we could show in a following study, in which we varied both the metal adatom as well as the work function of the substrate system. In all cases, the trend in observing certain charge states followed the above reasoning.

\section{Imaging Molecular Frontier Orbitals of Pentacene on NaCl Films}

As a bulk material pentacene is one of the best-studied organic semiconductors, having a relatively small transport-gap and a high charge carrier mobility. Therefore we chose individual pentacene molecules for our electron transport measurements on ultrathin $\mathrm{NaCl}$ films. ${ }^{[13]}$
Differential conductance $(\mathrm{d} I / \mathrm{d} V)$ spectroscopy on an individual pentacene molecule exhibits two distinct features in the $\mathrm{d} I / \mathrm{d} V$ signal, centered around -2.4 and 1.7 $\mathrm{V}$, and a broad gap region of low conductance between these peaks (Fig. 1). Biasdependent imaging shows that directly correlated to the spectroscopic features three qualitatively different STM images are obtained: When imaging within the broad gap region, the pentacene molecule appears as a featureless rod. In contrast, for voltages exceeding the peak positions, the molecule appears much broader and higher and exhibits internal structure (panels to the left and right). The spatial resolution can be further enhanced by picking up a pentacene molecule with the STM tip (lower row of STM images in Fig. 1). In this case, the image acquired at voltages in the gap region shows five faint protrusions, $2.3 \AA$ apart. The corresponding STM images at voltages below -2.4 and above $1.7 \mathrm{~V}$ very closely resemble the contours of constant probability density of the native highest occupied (HOMO) and lowest unoccupied molecular orbital (LUMO) of the free molecule (Fig. 2 and ref. [30]), which were calculated using the first-principles planewave density-functional-theory program DACAPO. [31]

We would like to mention that STM images with an intramolecular structure that resembles the LUMO of a molecule have also been obtained for molecules directly adsorbed on metal surfaces at high tunneling currents. ${ }^{[32-34]}$ However, the direct interpretation of these images in such a case is not straightforward. In the present case, tunneling at biases corresponding to a molecular resonance increases the tunneling current by about two orders of magnitude. This indicates that the through-molecule tunneling path clearly dominates tunneling at resonance, which also enables the direct imaging of the HOMO at negative bias voltage polarity. Only in conjunction with the clear, well-separated peaks in the $\mathrm{d} I / \mathrm{d} V$ spectroscopy can the bias-dependent STM images be unambiguously assigned to the molecular resonances. This technique can be applied to a wide range of different molecules. Apart from the mere possibility to obtain a direct image of molecular orbitals, we regard this as an important technique to unambiguously assign certain features in conductance spectroscopy. For example, the order of the peaks in the conductance spectra may deviate from the order of the molecular orbitals in its neutral state as a result of the temporary charging taking place when taking a spectrum. In other cases, there might be degeneracy between two electronic resonances that could be lifted by the environment. Also it could happen that several features in the conductance spectra relate to the same molecular orbital. In all of these cases, it will be important to assign the features to certain molecular orbitals, which in such a setup can simply be done by looking at the voltage-dependent images.

In the weak coupling regime, an electron or hole has to localize in the molecule before it can tunnel away into the substrate. Thus, the Coulomb energy $U$ for charging the molecule becomes important. The peak positions in the spectra correspond to the 
negative ion resonance (NIR) ${ }^{[35]}$ and positive ion-resonance (PIR) states of the adsorbed molecule, which have a larger energy spacing than the HOMO LUMO gap of the neutral molecule. These states are related to the ionization energy of $6.61 \mathrm{eV}^{[36,37]}$ and the electron affinity of $1.35 \mathrm{eV}^{[38]}$ of the free molecule. These energies are, however, shifted towards the Fermi level because of the electronic polarization of the $\mathrm{NaCl}$ film and the underlying metal.[39] The much slower ionic polarization of the substrate will take place only after the electron or hole is fully localized on the molecule, and therefore will not decrease the gap size.

In such a double-barrier tunneling junction in the weak coupling limit it is not obvious what fractions of the applied bias voltage drops over the vacuum and over the $\mathrm{NaCl}$ barrier, respectively. By varying the tip-molecule distance we could show that most of the voltage drops over the vacuum barrier and the remaining voltage drop over the insulator is only a few percent of the total voltage. ${ }^{[13,21]}$ By increasing the $\mathrm{NaCl}$ layer thickness, the electronic polarization of the metal substrate (image-charge effects) can be reduced and directly correlated to the observed width of the gap, which is 3.3, 4.1, and $4.4 \mathrm{eV}$ for one, two, and three $\mathrm{NaCl}$ layers, respectively. When changing the metal substrate orientation from $\mathrm{Cu}(111)$ to $\mathrm{Cu}(100)$, we observe an almost identical gap width for a bilayer of $\mathrm{NaCl}$, but an overall shift of the peak positions of $0.35 \mathrm{eV}$ towards lower voltages. This compares favourably with the difference in work functions $\Delta \Phi$, which for the clean surfaces is $\Delta \Phi=0.35 \mathrm{eV} .{ }^{[40]}$ This observation suggests that the molecular levels of the pentacene molecule are coupled to the vacuum level of the supporting $\mathrm{NaCl} / \mathrm{Cu}$ system.

\section{Phonon-induced Peak Broadening}

The conductance spectra discussed in the previous section show relatively broad peaks of full width at half maximum (FWHM) of roughly $0.3 \mathrm{eV}$. At first glance this is surprising, as the lifetime of an electron or hole in the molecule should be dramatically increased by the presence of the insulator. For two layers of $\mathrm{NaCl}$ the electronic coupling to the substrate should be so weak that an intrinsic linewidth on the order of few meV or even less could be expected.[13] The FWHM does not change from two to three layers of $\mathrm{NaCl}$, which also speaks against a lifetime broadening limited FWHM. The intrinsic energy resolution of elastic STS at $5 \mathrm{~K}$ is also in the range of only few meV. Therefore we investigated the peak broadening mechanism by analyzing dif-

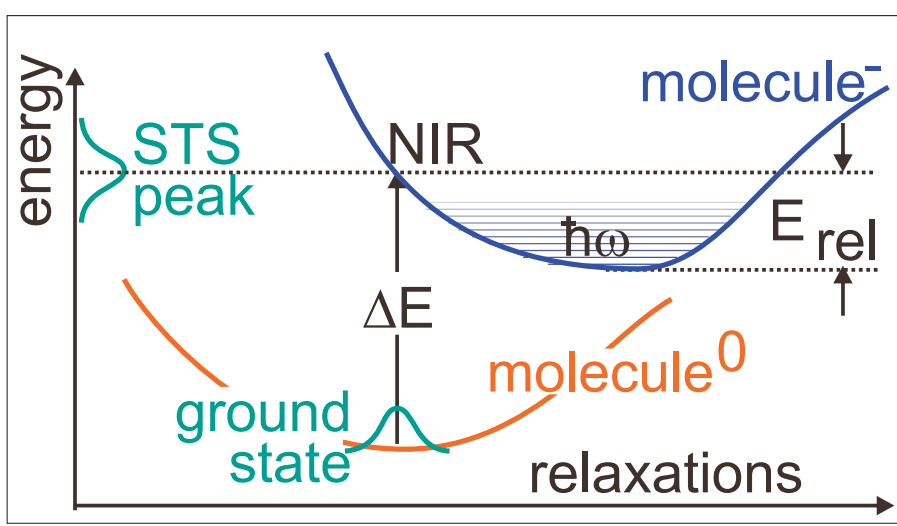

Fig. 3. Illustration of the broadening mechanism. Further explanations are given in the text.

ferential conductance spectra of localized states in Cl-vacancies in detail.[21] It turned out that the observed broad line shapes are caused by a strong coupling between the localized electronic state corresponding to the peak in the spectrum and the optical phonons in the film. The underlying physical mechanism can be understood from the Franck-Condon picture as depicted in Fig. 3 and described in the following. At resonance the electrons of the STS current temporarily charge the defect or adsorbate. Similar to the observations for the charge bistability the charged ions in the polar insulator react strongly to the charge. In the Franck-Condon picture this corresponds to a strong displacement of the potential minimum along the axis of ionic displacement. At low temperatures, the vertical electron attachment, repeatedly taking place when acquiring a spectrum, will encounter the system primarily in the phononic ground state. The slope of the potential at the negative ion resonance will translate the uncertainty in the positions of the ion cores in the ground state (zero-point motion) to an uncertainty of the energy of the negative ion resonance. ${ }^{[21,41,42]}$

To make a quantitative analysis, parameters were obtained from density functional calculations, in which the temporary occupation of the electronic state taking place in the experiment was accounted for. The resulting FWHM agrees well with the experimentally observed one. This simple model applies not only to the vacancies but to molecules as well. In addition, the simple model predicts a decreasing FWHM for different insulators in the order LiF, $\mathrm{NaCl}, \mathrm{RbI}, \mathrm{Xe}$. This prediction was confirmed in the case of pentacene molecules in corresponding experiments on all these films. ${ }^{[21]}$

These findings have strong implications for molecular electronics. If molecular electronics is to advance to more complex structures of connected molecular devices and not just a single molecule between two metallic leads, every molecular device will be embedded in a certain envi- ronment. As is described above, this environment may have dramatic effects on the conductance properties even if the environment is completely insulating. The strong phonon-broadening may also be used as an advantage. For example, it may help the electron transport between two levels that are not perfectly aligned.

\section{Contact Formation and Orbital Engineering}

The scanning probe techniques allow not only to image structures in real space on the atomic length scale, but also to position adsorbates and to create artificial structures with atomic precision. ${ }^{[43]}$ In combination with insulating films as substrates this in principle offers the prospect of creating nanoscale electronic devices that are electronically decoupled from their surrounding. ${ }^{[17]}$ It would be extremely interesting to build up a molecular device directly including the coupling leads with atomic precision. Such a control of the contact formation would allow selected coupling with respect to the molecular orbitals including their phase, which may be used to implement certain device functions. ${ }^{[44]}$ However, STM manipulation on insulators is qualitatively different from manipulation on metal surfaces and in particular the lateral placement of adsorbates is much more difficult. ${ }^{[17]}$

One first step in this direction is the formation of a covalent bond between a $\mathrm{Au}$ adatom and an individual pentacene molecule. ${ }^{[45]}$ A covalent bond formation between these two species is rather unexpected. Interestingly, the covalent bond involves the delocalized electronic orbitals of the pentacene molecule, facilitating a strong coupling of the electronic states that are involved in electron transport. Moreover, two different isomers of the same two constituents could be formed that show very different orbital structures. This 'engineering' of the orbital structure by creating different isomers controls both the nodal structure itself as well as the rela- 


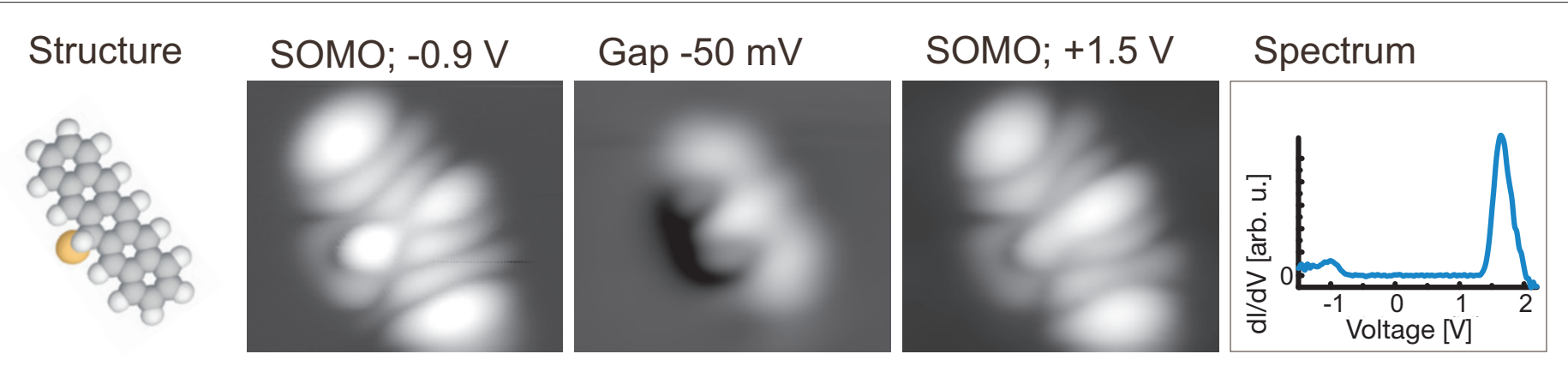

Fig. 4. Structural model, bias-dependent STM images and differential conductance (d//dV) spectrum of a 6-gold-pentacene complex formed out of a gold atom and a pentacene molecule on a $\mathrm{NaCl}$ film. The $\mathrm{d} / \mathrm{d} \mathrm{d}$ spectrum of the 6-gold-pentacene complex exhibits two distinct peaks at -1.2 and $+1.5 \mathrm{~V}$. At biases corresponding to the two peaks of the $\mathrm{d} / / \mathrm{d} V$ spectrum, the 6 -gold-pentacene complex yield STM images of the singly occupied molecular orbital and look similar.

tive weight of the probability distribution in different parts of the molecule.

One interesting side aspect of this work is that the formed complex has an odd number of electrons. This was experimentally confirmed by a charge determination as described at the end of Section 2. Consequently, the molecule has a singly occupied molecular orbital (SOMO) near the Fermi level. This SOMO gives rise to two peaks in conductance spectra at different bias voltage polarity. Images taken at voltages corresponding to the two peaks show the same orbital structure (Fig. 4). For negative or positive bias voltage the SOMO is temporarily emptied or filled, respectively. Thus, the broad gap in the $\mathrm{d} I / \mathrm{d} V$ spectra between those two peaks is not a HOMOLUMO gap, but is solely attributed to the Coulomb energy associated with adding or removing an electron to or from the same orbital of the complex. Thus, the separation of the two peaks labeled SOMO gives a simple and direct measure of this important parameter.

\section{Tautomerization-based Molecular Switch}

In this section we describe a molecular switch based on a tautomerization reaction. ${ }^{[46]}$ The switch consists of a freebase phthalocyanine or naphthalocyanine molecule and was studied in a double barrier junction in an experimental setup as described above for pentacene. These molecules host two hydrogen atoms in a central cavity. The hydrogens reduce the otherwise four-fold rotational symmetry $\left(D_{4 h}\right)$ of the molecular framework to a two-fold symmetry $\left(\mathrm{D}_{2 \mathrm{~h}}\right)$. Therefore, there are two equivalent possibilities for the positions of the two hydrogens. As in the case of pentacene, the frontier molecular orbitals can be directly imaged. The STM image corresponding to the LUMO has two-fold rotational symmetry. Its orientation therefore directly shows the position of the two hydrogens in the central cavity. The molecules can be switched in a controlled fashion between the two possibilities for the positions of the two hydrogens by an inelastic tunneling current. To induce this tautomerization reaction, the voltage has to be increased considerably beyond the voltage, at which the LUMO can be imaged (to about $1.4 \mathrm{~V}$ ). This implies that the position of the hydrogens can be 'read out' by taking an image of the LUMO without unintended switching. When the tip is placed outside the central region of the molecule, the tautomerization reaction is expressed as considerable changes in the conductance through the molecule, as can be seen in Fig. 5. The switching can not only be initiated by an inelastic current directly injected into the central region of the molecule, but also at the periphery of the molecule. This situation allows for an interesting two-probe experiment. The tautomerization reaction site is known to be localized to the very center of the mol-

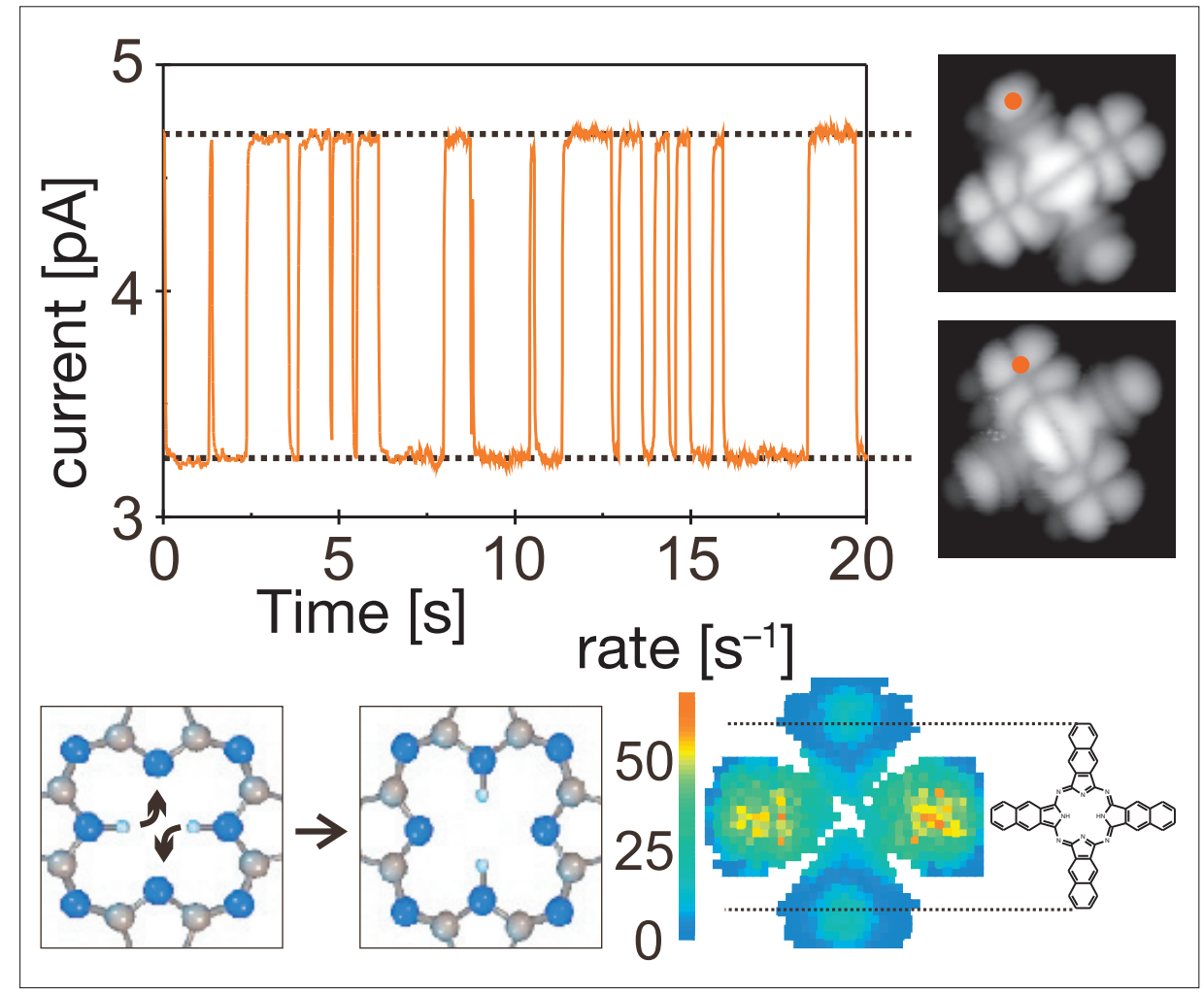

Fig. 5. Switching of a single naphthalocyanine molecule by the tunneling current. (Top-left) Current-trace obtained at a bias of $1.7 \mathrm{~V}$ when the tip was positioned at one end of the molecule (red dot in STM images). (Top-right) Orbital images showing the orientation of the LUMO corresponding to the high- or low-current state (2 pA, $0.7 \mathrm{~V}$ ). (Bottom-left) Schematic of the hydrogen tautomerization reaction responsible for the switching. (Bottom-right) Spatial map of the switching rate for the hydrogen tautomerization reaction for a tunneling current of $1 \mathrm{pA}$ at a bias of $1.825 \mathrm{~V}$. For reference, the structure of the molecule is displayed to scale. Adapted from ref. [46]. 
ecule, the injection of the current is also very local and determined by the tip position. The switching rate as a function of the position of current injection allows one to obtain direct information on the electron and/or energy transport within a single molecule in a different way than in usual conductance measurements. This was investigated in a statistical analysis and provided a direct spatial map of the switching yield. Interestingly this map shows distinct intramolecular resolution and strikingly the largest switching rate is achieved when the tip is above the far periphery of the molecule (i.e. $>10 \AA$ away from the reaction site).

Switching phenomena within individual molecules could potentially be used as nonvolatile memory with extremely high density, as has been proposed many times. ${ }^{[47-49]}$ In contrast to previously investigated systems, the present molecular switch is planar, does not involve conformational changes at the periphery of the molecule, and is well-suited for use in self-assembled monolayers. It is anticipated that the switching will also work with molecules embedded in all solid-state devices and in multicore porphyrin-class molecules acting as more complex devices. As a first step in this direction we also demonstrated a coupling of the switching process so that the charge injection in one molecule induced tautomerization in an adjacent molecule.

\section{Conclusion}

We addressed issues relevant to molecular electronics by STM and STS measurements of adsorbates on ultrathin insulating films. The ultrathin insulating films imply a weak coupling of the molecular levels to the leads, which are represented by the tip and substrate. It turned out that the insulating films play a much more important role than just providing an additional tunneling barrier. They also can lead to a self-stabilization mechanism of an additional charge in the adsorbate due to ionic relaxations in the insulator. This can result in a charge bistability as we observed for individual metal adatoms. In the case of molecules a charge bistability is less likely, but should still be possible. In the case of individual pentacene molecules on $\mathrm{NaCl} / \mathrm{Cu}(111)$, the electronic decoupling from the metal states brought about by the insulator opens up the unique possibility to directly image the apparently unperturbed molecular orbitals by means of STM and gaining insight into the electronic level alignment. The strong electron-phonon coupling that leads to the charge stabilization also leads to a strong broadening of the molecular resonances in conductance spectroscopy.
We used STM-based manipulation to form a covalent bond between a gold atom and an individual pentacene molecule. The organometallic bond formation transforms a closed-shell molecule into a radical. Different isomers could be created, by which a control of both the nodal structure itself as well as the relative weight of the probability distribution in different parts of the molecule could be demonstrated. Finally we employed the bistability in the position of the two hydrogen atoms in the inner cavity of single free-base naphthalocyanine molecules as a two-level molecular switch that could be manipulated and probed by the STM.

\section{Acknowledgments}

It is a pleasure to thank our collaborators Leo Gross, Fabian Mohn, Peter Liljeroth, Fredrik E. Olsson, Sami Paavilainen, Mats Persson, Sladjana Stoiković, and Christian Joachim. Valuable comments by Ingmar Swart and Rolf Allenspach and financial support by the Volkswagen Foundation through its Lichtenberg program, the DFG under the program SPP1243, the EU projects 'HERODOT', 'ARTIST', and the Swiss National Center of Competence in Research (NCCR) 'Nanoscale Science' are gratefully acknowledged.

Received: April 20, 2010

[1] N. Néel, J. Kröger, L. Limot, T. Frederiksen, M. Brandbyge, R. Berndt, Phys. Rev. Lett. 2007, 98, 065502

[2] G. Schulze, K.J. Franke, A. Gagliardi, G. Romano, C. S. Lin, A. L. Rosa, T. A. Niehaus, T. Frauenheim, A. Di Carlo, A. Pecchia, J. I. Pascual, Phys. Rev. Lett. 2008, 100, 136801.

[3] G. Schull, T. Frederiksen, M. Brandbyge, R. Berndt, Phys. Rev. Lett. 2009, 103, 206803.

[4] J. Libuda, F. Winkelmann, M. Bäumer, H. J. Freund, T. Bertams, N. Neddermeyer, K. Müller, Surf. Sci. 1994, 318, 61 .

[5] K. Glöckler, M. Sokolowski, A. Soukopp, E. Umbach, Phys. Rev. B 1996, 54, 7705.

[6] W. Hebenstreit, J. Redinger, Z. Horozova, M. Schmid, R. Podlucky, P. Varga, Surf. Sci. 1999, 424, L321.

[7] S. Schintke, S. Messerli, M. Pivetta, F. Patthey, L. Libioulle, M. Stengel, A. DeVita, W.-D. Schneider, Phys. Rev. Lett. 2001, 87, 276801.

[8] J. Repp, S. Fölsch, G. Meyer, K.-H. Rieder, Phys. Rev. Lett. 2001, 86, 252.

[9] J. Repp, G. Meyer, F. E. Olsson, M. Persson, Science 2004, 305, 493

[10] A. J. Heinrich, J. A. Gupta, C. P. Lutz, D. M. Eigler, Science 2004, 306, 466.

[11] X. H. Qiu, G. V. Nazin, W. Ho, Phys. Rev. Lett. 2004, 92, 206102.

[12] S. W. Wu, G. V. Nazin, X. Chen, X. H. Qiu, W. Ho, Phys. Rev. Lett. 2004, 93, 236802.

[13] J. Repp, G. Meyer, S. M. Stojković, A. Gourdon, C. Joachim, Phys. Rev. Lett. 2005, 94, 026803.

[14] N. Nilius, T. M. Wallis, W. Ho, Phys. Rev. Lett. 2003, 90, 046808

[15] N. Nilius, E. Rienks, H.-P. Rust, H.-J. Freund, Phys. Rev. Lett. 2005, 95, 066101

[16] H.-J. Freund, Surf. Sci. 2007, 601, 1438.

[17] C. F. Hirjibehedin, C. P. Lutz, A. J. Heinrich, Science 2006, 312, 1021.

[18] N. Tsukahara, K. Noto, M. Ohara, S. Shiraki, N. Takagi, Y. Takata, J. Miyawaki, M. Taguchi, A. Chainani, S. Shin, M. Kawai, Phys. Rev. Lett. 2009, 102, 167203 .
[19] Y.-S. Fu, S.-H. Ji, T. Zhang, X. Chen, X.-C. Ma, J.-F. Jia, Q.-K. Xue, Appl. Phys. Lett. 2009, 95, 063107.

[20] C. F. Hirjibehedin, C.-Y. Lin, A. F. Otte, M. Ternes, C. P. Lutz, B. A. Jones, A. J. Heinrich, Science 2007, 317, 1199.

[21] J. Repp, G. Meyer, S. Paavilainen, F. E. Olsson, M. Persson, Phys. Rev. Lett. 2005, 95, 225503.

[22] A. Hotzel, G. Moos, K. Ishioka, M. Wolf, G. Ertl, Appl. Phys. B 1999, 68, 615.

[23] J.-Y. Park U. D. Ham, S.-J. Kahng, Y. Kuk, K. Miyake, K. Hata, H. Shigekawa, Phys. Rev. B 2000, 62, R16 341

[24] M. F. Crommie, C. P. Lutz, D. M. Eigler, Nature 1993, 363, 524.

[25] Y. Hasegawa, P. Avouris, Phys. Rev. Lett. 1993, $71,1071$.

[26] F. E. Olsson, M. Persson, A. G. Borisov, J. P. Gauyacq, J. Lagoute, S. Fölsch, Phys. Rev. Lett. 2004, 93, 206803.

[27] L. Limot, E. Pehlke, J. Kröger, R. Berndt, Phys. Rev. Lett. 2005, 94, 036805.

[28] S. W. Wu, N. Ogawa, G. V. Nazin, W. Ho. $J$. Phys. Chem. C 2008, 112, 5241.

[29] L. Gross, F. Mohn, P. Liljeroth, J. Repp, F. J Giessibl, G. Meyer, Science 2009, 324, 1428

[30] R. G. Endres, C. Y. Fong, L. Yang, G. Witte, C. Wöll, Comp. Mat. Sci. 2004, 29, 362.

[31] DACAPO, version 2.6.1, CAMP, Technical University of Denmark, Lyngby, Denmark, 2002, using GGA(PW91), a $261713 \AA^{3}$ unit cell, $400 \mathrm{eV}$ plane-wave cut-off energy, and $0.05 \mathrm{eV} / \AA$ A residual forces, http://www.fysik.dtu. dk/campos.

[32] M. Böhringer, W.-D. Schneider, R. Berndt, Surf. Sci. 1998, 408, 72 .

[33] J. Lagoute, K. Kanisawa, S. Fölsch, Phys. Rev. B 2004, 70, 245415.

[34] W.-H. Soe, C. Manzano, A. De Sarkar, N Chandrasekhar, C. Joachim, Phys. Rev. Lett. 2009, 102, 176102 .

[35] The negative ion resonance is the negatively charged adsorbate with the system in the ionic configuration as for the neutral adsorbate.

[36] W. Schmidt, J. Chem. Phys. 1977, 66, 828.

[37] V. Coropceanu, M. Malagoli, D. A. da Silva Filho, N. E. Gruhn, T. G. Bill, J. L. Brédas, Phys. Rev. Lett. 2002, 89, 275503.

[38] L. Crocker, T. Wang, P. Kebarle, J. Am. Chem. Soc. 1993, 115, 7818.

[39] T. M. Halasinski, D. M. Hudgins, F. Salama, L. J. Allamandola, T. Bally, J. Phys. Chem. A 2000, 104, 7484 .

[40] P. O. Gartland, Phys. Norv. 1972, 6, 201.

[41] J. W. Gadzuk, Phys. Rev. B 1991, 44, 13466.

[42] N. S. Wingreen, K. W. Jacobsen, J. W. Wilkins, Phys. Rev. B 1989, 40, 11834.

[43] D. M. Eigler, E. K. Schweizer, Nature 1990 344, 524.

[44] A. Donarini, G. Begemann, M. Grifoni, Nano Lett. 2009, 9, 2897.

[45] J. Repp, G. Meyer, S. Paavilainen, F. E. Olsson, M. Persson, Science 2006, 312, 1196.

[46] P. Liljeroth, J. Repp, G. Meyer, Science 2007, 317,1203

[47] N. J. Tao, Nat. Nanotech. 2006, 1, 173.

[48] C. P. Collier, G. Mattersteig, E. W. Wong, Y. Luo, K. Beverly, J. Sampaio, F. M. Raymo, J. F. Stoddart, J. R. Heath, Science 2000, 289, 1172.

[49] A. Szuchmacher Blum, J. G. Kushmerick, D. P. Long, C. H. Patterson, J. C. Yang, J. C. Henderson, Y. Yao, J. M. Tour, R. Shashidhar, B. R. Ratna, Nat. Mater. 2005, 4, 167. 